\title{
COLLABORATIVE VOTING OF 3D FEATURES FOR ROBUST GESTURE ESTIMATION
}

\author{
Image Processing Group \\ Universitat Politècnica de Catalunya \\ Barcelona, Spain
}

Daniel van Sabben, Javier Ruiz-Hidalgo, Xavier Suau Cuadros and Josep R. Casas

\begin{abstract}
Human body analysis raises special interest because it enables a wide range of interactive applications. In this paper we present a gesture estimator that discriminates body poses in depth images. A novel collaborative method is proposed to learn $3 \mathrm{D}$ features of the human body and, later, to estimate specific gestures. The collaborative estimation framework is inspired by decision forests, where each selected point (anchor point) contributes to the estimation by casting votes. The main idea is to detect a body part by accumulating the inference of other trained body parts. The collaborative voting encodes the global context of human pose, while 3D features represent local appearance. Body parts contributing to the detection are interpreted as a voting process. Experimental results for different $3 \mathrm{D}$ features prove the validity of the proposed algorithm.
\end{abstract}

Index Terms - 3D features, body parts, skeleton joints localization, gesture estimation

\section{INTRODUCTION}

The detection of human features such as voice and gestures allows devices to respond in human detection applications. Over the past decade, new technologies have arisen to the point of enabling efficient human-machine interaction. This is the case of affordable depth sensors for computer vision. Leaving aside color images, depth data carry spatial information that may suit better geometrical measurements for space related detection. Additionally, advances in machine learning provide better computational models that adapt to training data. Improved data and classifiers allow for better detectors in estimation problems.

This paper focuses on the detection of particular configurations of the human body, providing relevant information as a strong indicator of the human behavior. Detecting body pose and gesture leads to outstanding applications in motion capture, human-computer interaction, improved surveillance, body-language interpretation, activity classification, sports monitoring, etc. The main contribution of this paper is a novel collaborative voting framework for depth images where full body pose and position of the body skeleton is jointly estimated.

The structure of the paper is as follows: the next section gives an overview of the state of the art in body pose estimation over depth data. Section 3 presents an overview of the proposed algorithm, while section 4 explains in detail the collaborative voting framework. The 3D features analyzed are explained in section 5. Finally, results and conclusions are drawn is sections 6 and 7 .

This work has been developed in the framework of project TEC201343935-R and TEC2016-75976-R, financed by the Spanish Ministerio de Economa y Competitividad and the European Regional Development Fund (ERDF).

\section{STATE OF THE ART}

Hough Forests for color images [1], as proposed by Gall et al [2], are a successful example for the detection of body parts. A hierarchical perspective of body parts is proposed by Navaratnam et al [3]. In the work presented by Eichner et al [4], an articulated human body model is used to improve the segmentation of body parts. More recently, Dantone et al [5] presented a double layered model for detecting body joints.

For depth information, the work of Shotton et al [6] trains a Random Forest to detect body parts. Although it requires a large training dataset (i.e. $+900 \mathrm{~K}$ images), the use of synthetic data is an interesting strategy to easily enlarge the dataset $[6,7]$. López et al [8] propose to detect specific body gestures by means of an unbalanced Random Forest approach. Their approach is largely real-time and robust, allowing frame-wise tracking of these gestures over time.

Other depth-based methods define an energy function specifically for depth data, eventually leading to impressive results $[9,10]$. In [10], a mixed Iterative Closest Point (ICP) which takes into account physical-spatial constrains is applied to modelled body parts. Schwarz et al [11] robustly detect anatomical landmarks in the 3D data and fit a skeleton body model using constrained inverse kinematics. Grest et al [12] use a non-linear least squares estimation based on silhouette edges able to track limbs in adverse background conditions. While many methods focus on upper-body pose, Plagemann et al [13] present a fast method which localizes body parts on 2.5D data at about 15 frames per second.

Closer to our proposal, Dantone et al [14] proposed a human pose estimation system using two-layered random forests as joint regressors. Similarly, Baak et al [15] proposed a solution where dataset samples are used to infer the current pose by looking for the best hypothesis that matches the current pose (based on a feature vector similarity). A generative method predicts the body pose and the final pose decision is determined by means of a voting process fusing both hypothesis components.



Fig. 1. General scheme. The functional block diagram used in both training and detection phases. 


\section{PROPOSED SCHEME}

We propose a discriminative scheme for body pose estimation. First, a training phase processes point clouds and extracts a collection of templates which characterize local parts of the body. Detection is done by processing the input cloud with the same (common) training scheme and, afterwards, its output is passed onto the Collaborative Voting algorithm which, using the previously trained templates, estimates a body pose.

The common scheme (Fig. 1) starts from a point cloud. In our case, the cloud is extracted from a Kinect depth camera, applying a plane clipping to remove background elements such as floor and walls. A point cloud subset is selected by random sampling as anchor points for further processing. After that, a 3D feature is computed from the anchor points neighborhood (Section 5 describes the features used). Finally, a data vector from every anchor is stored as training template.

In the detection phase, the common scheme first extracts the 3D features in a similar way, and a Collaborative Voting framework is then used to estimate the body pose together with the body joint positions.

\section{COLLABORATIVE VOTING FRAMEWORK}

The Collaborative Voting (CV) framework applies to multipart object detection. The Voting concept consists in inferring an object part location by an accumulation process, where each contribution can be counted as a vote, similarly to Hough accumulators. The word $\mathrm{Col}$ laborative comes from the idea that every object part cast votes to other object parts' locations, giving this sense of collaboration.

The proposed CV framework infers body joints locations in order to build a full body skeleton and a global pose ID identifying the current body gesture. Joints are found by accumulating votes from the cloud subset (anchors) contributing to joints locations (collaborative decision). Votes for each contribution are selected from templates trained based on anchors' local similarity.

In training, at the end of the common scheme, a data structure defined as training template is filled for every anchor point as shown in Fig. 2. A template is formed with: 3D anchor position, 1D feature histogram vector (see Section 5), difference vectors between body joints locations and anchor 3D position and a gesture ID number of the global pose. Note that the body joints locations are known from the groundtruth annotated in the training dataset.

In the detection phase, the same templates are filled for every anchor, but excluding the difference vectors and the pose gesture ID, which is the goal of the detection. A similarity measure is required for the detection process, as the algorithm has to select the most similar templates on the training template collection for a specific anchor. The distance between templates to find the more similar templates in the training template collection is as follows:

$$
\mathcal{D}_{i, k}=w \frac{1}{\frac{1}{N} \sum_{j \in N} s_{j}}\left|s_{i}-s_{k}\right|+(1-w)\left\|y_{i}-y_{k}\right\|^{2}
$$

This distance is a blend of 2 factors: the normalized anchor position $s$ for all training samples $N$ and the squared distance between $1 \mathrm{D}$ feature histograms $y$. A weighting parameter $w$ ranging from 0 to 1 is included to tune which of the 2 factors has more influence. Note that anchor position is normalized with the average anchor position of the training set samples $s_{j}$ to keep the same order of magnitude with the normalized 1D histogram distance.

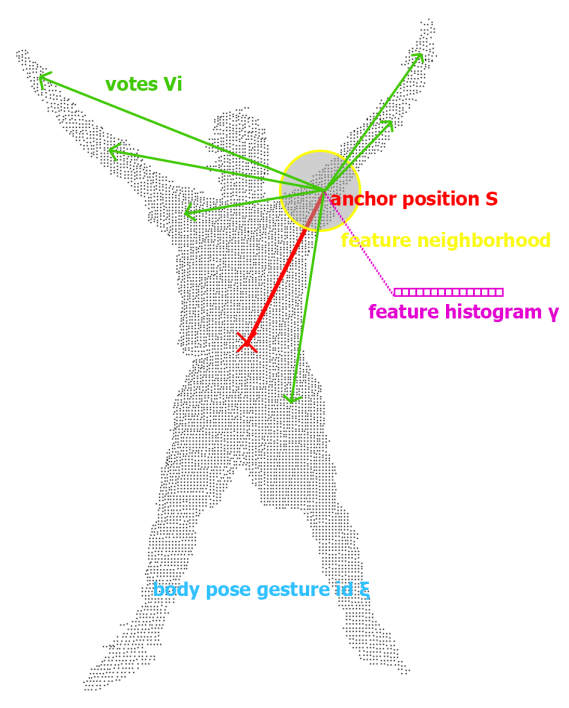

Fig. 2. Body anchor template components: 3D anchor position, neighboring points, votes to body joints, feature 1D histogram and body pose ID.

The voting process consists in accumulating votes from each anchor towards the overall detection. First, for every anchor, a k-NN is applied (using distance $\mathcal{D}_{i, k}$ ) on the training templates collection to obtain the most similar templates. A vote is defined as a 3D position hypothesis on where a specific body joint is located. In this context, votes are formed by adding the difference vectors (from the k-NN training templates) and the current anchor position being processed in detection. At this point, once all anchors have been processed, a collection of votes is retrieved. Next step is to accumulate all votes. For this purpose, a fixed 3D voxelization is proposed as the accumulator structure (votemap) for each body joint. The voting consists in incrementing the voxels value where a vote position falls into. The final estimated body joint location is the voxel position from its votemap with the maximum value. Note that with high voxelization resolution (small voxels) the voting process tends to increment isolated voxels and therefore leads to a non discriminative detection. To overcome this, a certain degree of smoothing is introduced by the influence of a Gaussian sphere around the votes. Fig. 3 shows an example of the Gaussian spheres voting for the hand position. Each vote increases the value of neighboring voxels around the vote position following a Gaussian decay distribution $I$ which is based on the distance between the voxel center $c$ and the vote position $v$. The variance parameter for this Gaussian decay is fixed to $12.5 \mathrm{~cm}^{2}$ :

$$
I=e^{-\frac{1}{12.5}\|c-v\|^{2}}
$$

The same idea is applied to detect the global pose ID but, instead of voxelizing, the accumulator is a 1D histogram with all possible body gestures as bins. In this case, a vote is an integer number which is the body gesture ID stored in the training template. Voting is done by increasing the histogram bin indicated by votes. Finally, the bin with maximum value is the detected global pose. 


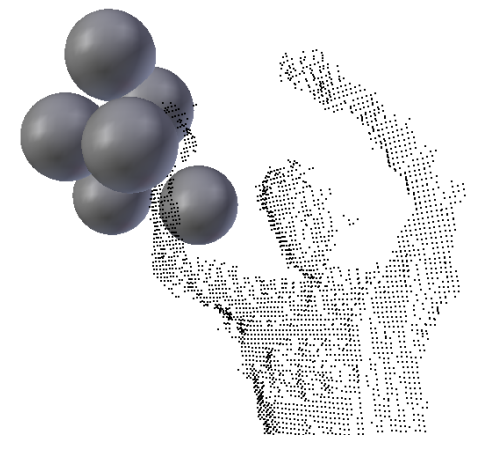

Fig. 3. Graphical representation of Gaussian sphere votes related to the right-hand joint.

\section{3D FEATURES}

This section describes the 3D features and the datasets used for the proposed collaborative voting framework.

We evaluate the collaborative voting with three different $3 \mathrm{D}$ features: Curvature, Oriented Radial Distribution and Histogram of Oriented Normal Vectors. These 3D features represent better the shape of point clouds than isolated points. Formally, the features are functions of a single point $p$ in the input cloud $\mathcal{P}$, coupled with featurespecific parameters $x=\left[x_{1}, \ldots, x_{n}\right]$ as its domain, and valued either as a scalar or a fixed dimension vector $y=\left[y_{1}, \ldots, y_{n}\right]$.

$$
y=f\left(N_{p, r}, x\right)
$$

where $N_{p, r}$ are the neighboring points where the feature is evaluated. These points are defined as points in $\mathcal{P}$ contained inside a sphere of radius $r$ and centered at $p: N_{p, r}=\left\{p_{0} \in \mathcal{P} \mid\left\|p_{0}-p\right\|<r\right\}$.

\subsection{Curvature}

Curvature $C$ is defined on the neighborhood $N_{p, r}$ as:

$$
C=\frac{\lambda_{0}}{\lambda_{0}+\lambda_{1}+\lambda_{2}}
$$

where $\lambda_{i}$ are the increasing eigenvalues of the covariance matrix of neighboring points location from Principal Component Analysis (PCA). In 3D, this measure indicates how much the smallest component differs from the other two. When $C$ equals zero, the points are contained in a plane. Curvature highlights the sparsity of non-planar point subsets.

\subsection{Oriented Radial Distribution}

Oriented Radial Distribution (ORD), as proposed by Suau et al [16], was designed to highlight prominent shapes in 3D space, i.e., point subsets considered as the part of a surface with non-homogeneous patterns. ORD is evaluated projecting the neighborhood onto its tangent plane and evaluating the homogeneity of its circular distribution. A specific filtering functionality is applied in low-density neighborhoods to avoid noise from depth sensors. ORD values for salient points in the surface are higher than those in planar regions.

\subsection{Histogram of Oriented Normal Vectors}

The Histogram of Oriented Normal Vectors $(H O N V)$ by Tang et al [17] capture local geometric characteristics from the normal vectors of surfaces. The authors propose a coarse normal estimation based on the image gradient, with depth variation as magnitude. For point clouds in 3D space, we compute normals from PCA analysis in a smaller neighborhood, choosing the smallest component as the normal.

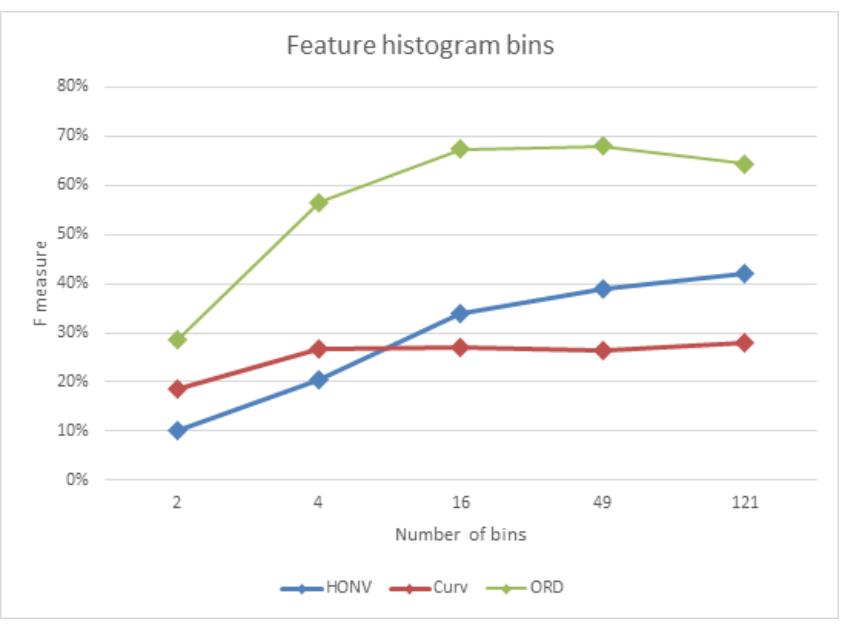

Fig. 4. Overall F-measure of HONV, Curvature and ORD with varying number of histogram bins

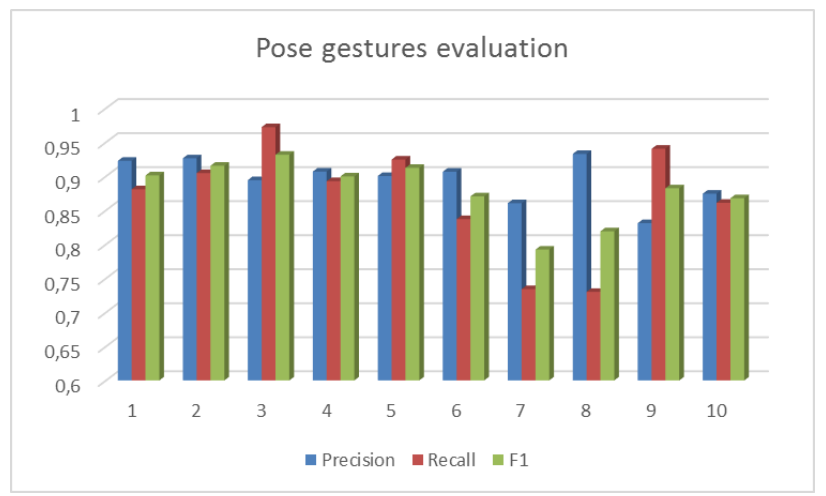

Fig. 5. Precision, Recall and F-measure for each pose gesture.

\section{RESULTS}

We evaluate our collaborative voting framework using two different datasets.

\subsection{Datasets and experiments}

Firstly, we introduce a publicly available dataset recorded using a Kinect camera at UPC [18]. The UPC dataset is composed of 12 recorded subjects performing 10 different standstill body poses (or static gestures) of different complexity. The groundtruth consists of 




Fig. 6. Average detection error in centimeters.

an associated label with the body pose and a frame-based articulated body model positions (14 joints).

Secondly, the Stanford dataset [19] is used to compare with other state-of-the-art methods.

In both datasets, a leave-one-out cross validation strategy is used. In all experiments, the parameters are set to 20 frames per gesture, neighbors radius $r=30 \mathrm{~cm}$, Gaussian deviation of $25 \mathrm{~cm}$ for creating the voting map and $K=15$ nearest neighbors per anchor and 25 .

\subsection{D Feature evaluation}

Fig. 4 assesses the behavior of the three 3D features varying the number of histogram bins. We set $w=0$ in Equation 1 so only the feature information is used as the distance metric. In the case of HONV, for which the histogram is $2 \mathrm{D}$, the number of bins is distributed equally in half to $\mathrm{X}$ bins and the rest to $\mathrm{Y}$ bins. The F-measure is used to evaluate the algorithm accumulating true/false and positives/negatives for the entire dataset.

ORD outperforms both Curvature and HONV in any configuration. All features tend to converge as the number of bins increase. In the ORD case, a late decay is observed due to overfitting. Consequently, values around $7 x 7=49$ bins are suitable for evaluation, considering the ORD has not decayed and the other two features are reaching convergence.

\subsection{Pose estimation evaluation}

Fig. 5 assesses the general body pose estimation accuracy. Results are extracted considering the best configuration for the detector (i.e. 49 histogram bins in the ORD feature, $w=0.5$ in Equation 1 and 50 random anchors in training). Fig. 5 shows the Precision, Recall and F-measure for each individual gesture. In general the system achieves a mean F-measure of 0.87 for the classification of the standstill body poses of the dataset.

Table 1 shows the average Euclidean distance between the estimated joint position of our proposed method and groundtruth for the $U P C$ dataset.

Fig. 6 compares the collaborative voting framework with [10] using the Stanford dataset. [10] obtains an average error of $7.3 \mathrm{~cm}$ while our method obtains an average error of about $9.7 \mathrm{~cm}$. We remind the work in [10] is fully dedicated to human body, incorporating a $3 \mathrm{D}$ model to fulfill these requirements. Moreover, the Stan-
Table 1. Average distance error associated with articulations

\begin{tabular}{lr||lr} 
Joint & Error & Joint & Error \\
\hline Head & $5.7 \mathrm{~cm}$ & Neck & $4.9 \mathrm{~cm}$ \\
Left Shoulder & $4.8 \mathrm{~cm}$ & Right Shoulder & $5.2 \mathrm{~cm}$ \\
Left Elbow & $12.1 \mathrm{~cm}$ & Right Elbow & $10.9 \mathrm{~cm}$ \\
Left Hand & $20.2 \mathrm{~cm}$ & Right Hand & $17.1 \mathrm{~cm}$ \\
Left Hip & $5.7 \mathrm{~cm}$ & Right Hip & $6.6 \mathrm{~cm}$ \\
Left Knee & $4.4 \mathrm{~cm}$ & Right Knee & $5.8 \mathrm{~cm}$ \\
Left Foot & $5.4 \mathrm{~cm}$ & Right Foot & $7.8 \mathrm{~cm}$
\end{tabular}

ford dataset is composed of 28 different sequences. Therefore, it is difficult to find training examples similar to the test ones, since we are using the complementary sequences as training for a given test sequence. Despite this inconvenient setup, the proposed Voting approach manages to select appropriate training templates, achieving good classification results with a generalized object parts detector.

\section{CONCLUSIONS}

After testing our proposed solution with the best configuration found we achieve a $0.87 \mathrm{~F}$-score average on body gesture classification and an average articulation error of $9.7 \mathrm{~cm}$ on body skeleton estimation.

These results are competitive to current state-of-the-art techniques. Taking into account that the Collaborative Voting framework introduced in this paper exhibits a generic behavior and could be applied directly to other detection problems, we consider that the obtained results contribute positively in this scenario.

Furthermore, the proposed technique is able to estimate, at the same time, both the full body pose and the position of the body skeleton. As future work, we are investigating the use of the Collaborative Voting framework in other applications, such as hand detection and to incorporate other priors, such as color information, into the voting framework.

As future work, we are investigating the use of the Collaborative Voting framework in other applications, such as hand detection and to incorporate other priors, such as color information, into the voting framework 


\section{REFERENCES}

[1] M. Van den Bergh, E. Koller-Meier, and L. Van Gool, "Realtime body pose recognition using $2 \mathrm{~d}$ or $3 \mathrm{~d}$ haarlets," International Journal of Computer Vision, vol. 83, no. 1, pp. 72-84, 2009.

[2] J. Gall, A. Yao, N. Razavi, L. Van Gool, and V. Lempitsky, "Hough forests for object detection, tracking, and action recognition," IEEE Transactions on Pattern Analysis and Machine Intelligence, vol. 33, no. 11, pp. 2188-2202, 2011.

[3] R. Navaratnam, A. Thayananthan, P. Torr, and R. Cipolla, "Hierarchical part-based human body pose estimation," in British Machine Vision Conference, 2005, vol. 1, pp. 479-488.

[4] M. Eichner, M. Marin-Jimenez, A. Zisserman, and V. Ferrari, " $2 \mathrm{~d}$ articulated human pose estimation and retrieval in (almost) unconstrained still images," International Journal of Computer Vision, vol. 99, no. 2, pp. 190-214, 2012.

[5] M. Dantone, J. Gall, C. Leistner, and L. Van Gool, "Human pose estimation using using body parts dependent joint regressors," in Computer Vision and Pattern Recognition, 2013, vol. 1, pp. 3041-3048.

[6] J. Shotton, R. B. Girshick, A. W. Fitzgibbon, T. Sharp, M. Cook, M. Finocchio, R. Moore, P. Kohli, A. Criminisi, A. Kipman, and A. Blake, "Efficient human pose estimation from single depth images," IEEE Transactions on Pattern Analysis and Machine Intelligence, vol. 35, no. 12, pp. 28212840, 2013.

[7] C. Keskin, F. Kiraç, Y. E. Kara, and L. Akarun, "Hand pose estimation and hand shape classification using multi-layered randomized decision forests," in European Conference in Computer Vision, 2012, pp. 852-863.

[8] A. López-Méndez and J. R. Casas, "Can our TV robustly understand human gestures? real-time gesture localization on range data," in European Conference on Visual Media Production, 2012, pp. 18-25.

[9] M. Siddiqui and G. Medioni, "Human pose estimation from a single view point, real-time range sensor," in Computer Vision and Pattern Recognition Workshops, 2010, pp. 1-8.

[10] V. Ganapathi, C. Plagemann, D. Koller, and S. Thrun, "Realtime human pose tracking from range data," in European Conference in Computer Vision, 2012, pp. 738-751.

[11] L. A. Schwarz, A. Mkhitaryan, D. Mateus, and N. Navab, "Human skeleton tracking from depth data using geodesic distances and optical flow," Image and Vision Computing, vol. 30, no. 3, pp. 217-226, 2012.

[12] D. Grest, V. Krüger, and R. Koc, "Single view motion tracking by depth and silhouette information," Lecture Notes in Computer Science, vol. 4522, pp. 719-729, 2007.

[13] C. Plagemann, V. Ganapathi, D. Koller, and S. Thrun, "Realtime identification and localization of body parts from depth images," in IEEE International Conference on Robotics and Automation, 2010, pp. 3108-3113.

[14] M. Dantone, J. Gall, C. Leistner, and L.V. Gool, "Human pose estimation using body parts dependent joint regressors," in IEEE Conference on Computer Vision and Pattern Recognition, 2013, pp. 3041-3048.
[15] A. Baak, M. Müller, G. Bharaj, H. Seidel, and C. Theobalt, "A data-driven approach for real-time full body pose reconstruction from a depth camera," in Consumer Depth Cameras for Computer Vision, pp. 71-98. Springer London, 2013.

[16] X. Suau, J. Ruiz-Hidalgo, and J. R. Casas, “Oriented radial distribution on depth data: application to the detection of endeffectors," in IEEE International Conference on Acoustics, Speech and Signal Processing, 2012, pp. 789-792.

[17] S. Tang, X. Wang, X. Lv, T. X. Han, J. Keller, Z. He, M. Skubic, and S. Lao, "Histogram of oriented normal vectors for object recognition with a depth sensor," in Asian Conference in Computer Vision, 2013, pp. 525-538.

[18] D. Van Sabben, A. Gil, and J. Ruiz-Hidalgo, "Human body pose dataset, https://imatge.upc.edu/web/resources/body-posedataset," January 2016.

[19] V. Ganapathi, C. Plagemann, D. Koller, and S. Thrun, "Real time motion capture using a single time-of-flight camera," in IEEE Conference on Computer Vision and Pattern Recognition, 2010, pp. 755-762. 